\title{
Reflexiones sobre los propósitos de un curso de matemáticas de primeros semestres en la educación superior en Colombia.
}

\author{
EDGAR A. BARÓN POVEDA. \\ HUGO E. ZAMORA CORONADO. \\ eabaronp@poligran.edu.co \\ hzamora@poligran.edu.co
}

\section{RESUMEN}

Los supuestos que la Educación Superior en Colombia asume respecto de la formación básica que en matemáticas deben poseer los aspirantes a ingresar en sus aulas, distan de la realidad intelectual del estudiante promedio.

La universidad ha hecho intentos, - usualmente coyunturales -, por subsanar las carencias de los estudiantes, pero en general no se han alcanzado los propósitos de nivelar al estudiante a las exigencias del ente superior. Se propone una visión de carácter estructural de la problemática, con objeto de lograr que un estudiante de primeros semestres reelabore comprensivamente una noción de matemáticas y por consiguiente, soporte adecuadamente los procesos de abstracción y generalización que le propone el plan de estudios de su carrera.

\section{PALABRAS CLAVE}

Aprendizaje, actividad de aprendizaje, didáctica de las matemáticas,.

\section{ABSTRACT}

The assumptions that Higher Education in Colombia assume with respect to the basic formation that the candidates must have in mathematics when entering the classrooms are away from the intellectual reality of the average student.

The university has tried - usually circumstantially - to repair the students' lacks; however, in general the objectives have not been reached with respect to the terms of placement of the students regarding the requirements of the institution.

We propose a structural vision of the issue in order to make a freshman learn comprehensively a notion of maths. Therefore, this student will be able to support correctly the abstract and general processes proposed by the plan of studies of his/ her career.

\section{KEY WORDS}

Learning, Didactics of Mathematics, Learning Activity.

\section{Edgar Alberto Barón Poveda.}

Licenciado en Matemáticas con Especialización en Gerencia de Mercadeo y Docencia Universitaria. Coordinador de Área del Departamento de Matemáticas. Ha publicado los libros: Matemáticas I y Matemáticas Básicas para Mercadeo y Publicidad. (Bogotá: Politécnico Grancolombiano, 2003. Actualmente también se desempeña como profesor en Educación Distribuida.

Sus intereses investigativos giran en torno al aprendizaje de las Matemáticas desde la perspectiva de construcción de conocimiento y las Matemáticas de la teoría de la Elección.

\section{Hugo Edver Zamora Coronado.}

Licenciado en Ciencias de la Educación con especialidad en Matemáticas. Especialización en Educación a Distancia. Docente del Departamento de Matemáticas del Politécnico Grancolombiano. Intereses investigativos en torno al aprendizaje por construcción; en particular, el relativo al conocimiento matemático escolar. 


\section{ACERCA DE LA FORMACIÓN MATEMÁTICA EN LA EDUCACIÓN SUPERIOR.}

La formación matemática que se proponga a un estudiante de la educación superior, debe ajustarse a las exigencias de una sociedad en evolución constante y que requiere de respuestas eficaces a los interrogantes que plantea a sus integrantes. Por lo tanto, sin dejar de lado los contenidos tradicionales de las matemáticas, que han hecho parte de los planes de estudio de la escolaridad superior, es necesario que el desarrollo de habilidades y capacidades matemáticas esenciales sea explícito en los procesos escolares y se convierta en foco de atención y realización de las labores de difusión del conocimiento matemático.

Entre las destrezas que se deben desarrollar en un ciudadano en formación cabe señalar: el planteamiento y solución de problemas, el desarrollo de pensamiento y razonamiento matemático, la simbolización y argumentación, la modelización y representación, y el establecimiento de conexiones con otras áreas del conocimiento.

Para alcanzar una visión acerca de las maneras como la Educación Superior puede responder a los desafíos respecto de la formación matemática de un egresado de sus aulas, es pertinente reconocer diversos puntos de vista acerca de concepciones de la actividad matemática. Una de tales visiones la provee Miguel de Guzmán cuando afirma que " La matemática es una exploración de ciertas estructuras complejas de la realidad que, mediante un proceso de simbolización adecuado de los objetos a los que se acerca y mediante una manipulación racional rigurosa de ellos, se dirige hacia un dominio efectivo de dicha realidad”. (De Guzmán, 1998, Pág. 332).

El estudio de diversas formas de la complejidad de la realidad, según el mismo De Guzmán, corresponde al trabajo histórico de la matemática. Basta mirar las aproximaciones tradicionales a la cantidad y la forma que dieron origen a la aritmética y a la geometría. La atención sistemática que el individuo dirige hacia diversas formas de la realidad, permite que otras áreas del conocimiento matemático sean desarrolladas: el álgebra, la probabilidad, la lógica entre otras.

Desde esta perspectiva, cualquier individuo debe enfrentar la complejidad de la realidad, como un requisito para ejercer dentro de la sociedad. El sistema escolar ha sido encargado por la sociedad de proponer acciones que po- sibiliten en el individuo comprensiones y elaboraciones acerca de dicha realidad. Las matemáticas de la escolaridad se convierten en el espacio natural donde se acerca al ciudadano en formación, a la reflexión sobre los elementos de la realidad física o mental de la que habla De Guzmán. Por lo tanto, el sistema escolar y en particular la Educación Superior deben proveer al estudiante de una formación de calidad que lo habilite para confrontar los retos que la realidad impone, sin importar la complejidad de dicha realidad.

Otra concepción del significado de la actividad matemática muestra el carácter amplio de ella, cuando se señala que: "La matemática es una ciencia exploratoria que busca comprender cualquier tipo de patrón, patrones que ocurren en la naturaleza, patrones inventados por la mente humana e incluso patrones derivados de otros patrones."(Steen, 1999, pág. 35). Luego, su estudio en la escolaridad debe constituirse en una experiencia de amplio recorrido sobre la cotidianidad física o mental del individuo, con objeto de caracterizar y lograr comprensiones sobre regularidades, variaciones y situaciones afines a los patrones.

En suma, la experiencia matemática que se viva en la Educación Superior debe contribuir en la formación del profesional, a la comprensión de mundos sometidos a fluctuaciones que no necesariamente obedecen a comportamientos señalados por la tradición o por la historia de la evolución de las ideas.

\section{PROBLEMÁTICA DE LOS CURSOS DE MATE- MÁTICAS EN LOS PRIMEROS SEMESTRES DE PROGRAMAS DE FORMACIÓN PROFE- SIONAL}

La formación matemática en la educación superior en Colombia está sometida a tensiones propias de supuestos que se hacen respecto de la formación básica de los estudiantes que ingresan a este nivel de formación y de exigencias respecto de la calidad de los egresados del sistema.

En cuanto a los supuestos, es usual asumir que un estudiante que ingresa a la universidad cuenta con conocimientos disciplinares mínimos y con herramientas intelectuales básicas que lo habilitan para abordar los procesos de desarrollo de pensamiento que le plantea su nueva experiencia escolar. Sin embargo, la realidad parece contradecir 
este supuesto en cuanto se refieren experiencias de docentes de matemática que caracterizan la formación del estudiante que ingresa.

Un estudio en la Universidad del Valle señala que al mismo tiempo con estudiantes de buena formación matemática, se encuentran en el primer semestre de Ingeniería, estudiantes que por su deficiente formación matemática corren el riesgo de abandonar la universidad o permanecer en ella durante lapsos a todas luces de inconveniencia social. (Robledo, 2005). Una encuesta a profesores de la Universidad Nacional de Medellín señalaba, en 1999, la seria preocupación por la "deficiente formación matemática en primaria y secundaria" que redunda en los resultados de los estudiantes y esto "a pesar de los filtros del examen de admisión" (Cossio, 1999). Un artículo que "realiza reflexiones sobre las causas del bajo aprovechamiento estudiantil en el curso de Matemáticas 1" (Posso, 2005), afirma "que el problema no es único de esta universidad" y lo caracteriza en términos de la diferente formación matemática de los recién ingresados como producto de la diferente cantidad de horas semanales de dedicación a matemáticas en sus colegios.

La problemática es de alta sensibilidad en los medios académicos a tal punto que en el año 2005 en la Universidad ICESI de Cali, se desarrolló el evento "Matemáticas: Del bachillerato a la universidad", cuyo objetivo general expresa: "Propiciar un espacio en el cual los responsables del área de Matemáticas, de nivel universitario y de educación básica y media, compartan experiencias, inquietudes y propuestas relacionadas con el desempeño académico y el nivel de conocimientos y competencias en matemáticas, de los estudiantes del primer semestre universitario, frente a los estándares básicos de calidad en matemáticas para la secundaria establecidos por el MEN".

Actualmente, las exigencias respecto de la calidad de la formación de los egresados de la educación superior, se describen en términos de una inserción efectiva del nuevo profesional en el entorno laboral. Descripciones de este orden se denominan competencias y muchas de ellas se relacionan con la formación matemática del egresado. Entre otras se indican: capacidad de análisis y síntesis, toma de decisiones, habilidad para trabajar en forma autónoma, resolución de problemas.

Estas declaraciones que tienden a convertirse en están- dares regionales (Europa) con posibilidad de expansión mundial (Tuning, 2003), apenas comienzan a permear la universidad latinoamericana y causan controversia respecto de su impacto en la difusión del conocimiento en la universitadad, pues no tienen aún el respaldo de los gobiernos del área y por tanto, no se han convertido en puntos de referencia del quehacer académico (Tuning América Latina, 2007). Sin embargo, en espacios académicos empieza la discusión acerca de los significados de currículos por competencias, discusión que de hecho problematiza también los entornos escolares, donde se orientan procesos relacionados con la actividad matemática.

La problemática que concurre en el aula de la clase de matemáticas de los primeros semestres, es compleja, pues su actividad se ve influida por las experiencias previas de los estudiantes, pero a la vez debe influir en la formación que empieza a asumir dicho estudiante. Las soluciones propuestas por las instituciones universitarias, con el fin de posibilitar que los estudiantes adquieran los conocimientos matemáticos necesarios para cursar una primera asignatura de matemáticas, en general plantean:

-La formulación y desarrollo de cursos cortos, previos al ingreso del estudiante a las aulas donde se efectúa "un repaso" de los contenidos que requiere el estudiante como anteriores al primer curso de matemáticas.

-La participación del estudiante en un curso semestral "anterior" al primer curso regular de matemáticas donde se "logre la nivelación" del estudiante, es decir donde el estudiante demuestre dominio sobre nociones previas al curso.

-El uso de cursos virtuales, que permitan una aproximación a los requerimientos mínimos para cursar la primera asignatura de matemáticas, en un programa de estudios.

-El manejo de una parte del tiempo que se asigna a una asignatura de matemáticas, para "revisar" los conocimientos anteriores que son requisito para el curso que se toma.

-La utilización de apoyos pedagógicos (monitorías, asesorías, etcétera) que ayuden al estudiante a identificar sus carencias en términos de conocimientos necesarios para el curso y le oriente sobre posibles formas de acceso a estos conocimientos.

Estos intentos de solución son propios de programas de estudio donde las matemáticas son esenciales en el pro- 
ceso de formación del profesional, como es el caso de las carreras de ciencias básicas y las ingenierías. Los resultados no parecen ser los esperados, pues docentes de cursos posteriores a un primer curso de matemáticas, perciben que la formación matemática básica de los estudiantes no se acerca a las cada vez mayores exigencias intelectuales que imponen los procesos de desarrollo de pensamiento en cuanto a mayor generalización y abstracción.

En programas donde las matemáticas desempeñan un papel de menor relevancia en la formación, se han asumido parcialmente algunas de las soluciones que se han descrito, o se han tomado caminos que podrían calificarse de extremos. Tal es la situación de programas de estudios que reducen sensiblemente el número de asignaturas de matemáticas que tradicionalmente hacían parte de sus mallas curriculares, o de programas que proponen cursos de matemáticas cuyos contenidos son los mismos de la Educación Básica, o aún programas que consideran factible prescindir de materias de matemáticas en sus planes de estudios.

Un problema adicional parece sumarse a los anteriormente referidos: se considera en varios entornos escolares que la política mundial de cobertura en educación afecta la calidad de la misma, y en particular, la que tiene que ver con la formación en matemáticas. Hasta hace pocos años el sistema de educación superior se caracterizó por la selectividad de sus estudiantes. Rigurosos exámenes de selección garantizaban que a sus "aulas" únicamente ingresaran los mejores egresados de la educación básica y media (esto sigue siendo cierto en muchas de nuestras universidades). Por lo tanto, el supuesto de la buena formación básica de los nuevos estudiantes no tenía discusión. El examen de admisión cumplía sus propósitos.

Pero, las nuevas concepciones alrededor de una sociedad que funda sus quehaceres en el conocimiento, exigen acciones coherentes en el sistema escolar. Por lo tanto, se pregona el acceso a la educación superior por parte de sectores amplios de la población, como uno de los soportes de dichas concepciones. Las cifras mundiales, regionales o locales lo corroboran. En el mundo, entre el año 1990 y el 2005 el número de estudiantes matriculados en programas universitarios de pregrado se incrementó en $48 \%$ aproximadamente. En América Latina esta cifra es cercana al $43 \%$ y en Colombia es del orden del $40 \%$. (EFA,
2008). Un factor que ha incidido en este repunte es el relacionado con los subsidios estatales para estudiar, o con las facilidades que ofrece el sector financiero para adelantar estudios superiores. Al margen vale la pena anotar, que a pesar de estas cifras positivas, el sistema de educación superior en el mundo apenas acoge (al término del año 2005 ) en promedio al $24 \%$ de la población en posibilidad de acceder a sus aulas.

Para el caso de Colombia, es común la apreciación de que el volumen de estudiantes que ingresa a las aulas universitarias no llena las expectativas del sistema en cuanto a requisitos mínimos de ingreso. Se menciona que esta "universalización" de la educación superior ha contribuido a acentuar problemáticas de adaptación y permanencia dentro de la institución universitaria y que la situación en cursos de matemáticas es todavía más álgida pues los porcentajes de deserción o pérdida de primeros cursos son los más elevados.

Los interrogantes respecto de la formación matemática de los futuros profesionales que requiere el país, saltan a la vista. ¿Cómo posibilitar que los estudiantes que ingresan a las aulas universitarias alcancen niveles de desarrollo de pensamiento compatibles con las exigencias sociales en términos de formación? ¿Cómo asumir el reto que se plantea a la universidad respecto de la diversa formación matemática que tienen los recién ingresados a las aulas y de la necesidad de alcanzar niveles mínimos de conocimientos, destrezas y competencias en matemáticas, que les permita abordar procesos de formación básica en matemáticas? Cómo plantear y ejecutar acciones que promuevan en el estudiante que ingresa a la universidad una cultura de esfuerzo intelectual como base de su proceso de aproximación al conocimiento en términos de formación matemática? ¿Cómo conciliar las políticas de cobertura y culminación de procesos formativos en educación con las políticas de calidad en la formación matemática de un egresado de la educación superior?

\section{CONCEPTUALIZACIÓN DE UNA ACTIVIDAD DE REELABORACIÓN DE UNA NOCIÓN EN MATEMÁTICAS.}

Las respuestas a la problemática descrita - lo que parece suceder en los escenarios de los cursos de matemáticas de primeros semestres en la universidad - se han caracteriza- 
do por un manejo desde la tradición de la enseñanza de la matemática en el nivel superior.

Se parte del supuesto de que la educación básica ha asumido su trabajo con las matemáticas escolares en términos de procesos tales como: acercar a los estudiantes a la manipulación de objetos matemáticos, por ejemplo, los conjuntos numéricos y las operaciones entre sus elementos; o aproximar la identificación y uso de propiedades comunes que subyacen a la manipulación referida; por ejemplo, las propiedades de las operaciones.

Otro supuesto asume que en términos de destrezas y competencias se ha hecho una labor con los llamados procesos básicos de aprendizaje en matemáticas: resolución de problemas, razonamiento y argumentación, comunicación, modelación (Lineamientos curriculares, 1994).

Por lo tanto, se infiere que el desarrollo de un curso de matemáticas de primeros semestres en la universidad se centra en la identificación y usos de estructuras que cobijan los objetos de estudio en las matemáticas de la educación básica y media. El acercamiento a estas estructuras y sus propiedades, debe entonces ser el camino que posibilite un desarrollo de pensamiento de mejor nivel de elaboración y abstracción, con las consecuencias sobre un mayor desarrollo de pensamiento.

Desde es perspectiva, las propuestas de "nivelación" en matemáticas, se centran en un recorrido por los contenidos disciplinares de la educación básica, en una intensidad variable como se describió en otro aparte. La esperanza implícita del planteamiento es, que la revisión que se hace a las temáticas, ayuda a superar carencias, llenar "vacíos", y provee una metodología de estudio en matemáticas. La realidad supera claramente las expectativas que se generan con estos intentos, pues los supuestos respecto del dominio de los aspectos manipulativos de una noción, o del reconocimiento y uso de aspectos comunes a las nociones, no hacen parte del bagaje intelectual del estudiante promedio que en la actualidad accede a las aulas universitarias.

El dilema que se suscita es complejo: por una parte, no es posible considerar que la manera de recuperar al estudiante para la experiencia matemática, sea plantearle el devol- verse a realizar el trabajo propio de la escolaridad básica, ya que en términos del tiempo de permanencia aceptable en el ente universitario, generaría una problemática; pero por otra parte, plantear un trabajo de recuperación sin considerar a fondo los diversos aspectos que se deben dominar de una noción en matemáticas, se constituye en una "pérdida" de tiempo dentro del proceso de formación profesional.

Entonces, hay que considerar otros elementos que ayuden a dilucidar un curso de acción que contribuya al manejo de la problemática identificada y que consulten la realidad del estudiante, del docente y del sistema escolar.

-Las propuestas de recuperación para la experiencia matemática de un estudiante que ingresa a las aulas universitarias, deben sustentarse en la reelaboración de una noción. Con este término se quiere señalar que hay que reconocer que el estudiante tiene en su haber algún tipo de conocimiento relacionado con una noción en estudio, en particular si esta es una noción que se declara como propia de los procesos de la escolaridad básica o media en matemáticas. Por lo tanto, hay que adelantar acciones o proponer actividades que permitan identificar los procedimientos y las concepciones que subyacen a estos procesos, cuando un estudiante trabaja en situaciones relacionadas con una noción.

A este respecto, se deben generar experiencias que coloquen al estudiante en situación de proponer formas de solución a interrogantes relacionados con la noción y que lo conminen a revisar, reconstruir y comunicar dichos procedimientos. Es importante que el estudiante declare las justificaciones que desde su mirada soportan los procedimientos asumidos. De aquí se debe posibilitar un trabajo de confrontación y validación de lo que cree que conoce el estudiante sobre la noción estudiada.

La reelaboración de la noción se refiere entonces al camino que en conjunto con el docente, recorra el estudiante para alcanzar comprensiones de los elementos que constituyen la noción en estudio, y por lo tanto, lograr elaboraciones con dicha noción en contextos donde esta accione. La actividad o actividades de reelaboración señalan acciones por desarrollar - en espacios tanto en el aula o fuera de ella, físicos o virtuales - para cumplir los propósitos de la reelaboración de la noción. 
Aquí es fundamental la responsabilidad que asuma el estudiante respecto del proceso de reelaboración anunciado. Esto es, se precisa de un compromiso individual, decidido y continuo del estudiante para transitar este camino de reelaboración propuesta. La transformación intelectual es una decisión del estudiante, y por tanto, un acto de la razón (Gallego, 1992).

- Proponer actividades en el camino de reelaboración de una noción, plantea el interrogante acerca de las concepciones que soportan las actividades. Señalar que la tradición de la enseñanza en educación superior enmarca los intentos de solución de la problemática respecto de la formación básica en matemáticas, significa que la docencia en matemáticas en educación superior se ejerce en analogía con la matemática como conocimiento lógico deductivo, o mejor aún intentando emular el trabajo del matemático que prefiere "confiar en sus concepciones estructurales, aunque también utiliza las procedimentales" (Kieran, 1992). Esta visión sin duda es procedente en cursos de nivel superior y con estudiantes que poseen conocimientos disciplinares básicos y procesos de pensamiento adecuados a la exigencia intelectual del nuevo conocimiento.

Sin embargo, en los cursos de matemáticas de primeros semestres de un programa de estudios universitarios, vale la pena examinar la pertinencia de un ejercicio docente que acentúa su trabajo en la concepción estructural de la noción, y en cambio considera que la concepción procedimental de la misma es superflua o poco importante de cara al logro de propósitos señalados para el curso. Pero como la misma autora Kieran señala, "..se ha mostrado que tanto la enseñanza como el contenido enfatizan las consideraciones estructurales y que los estudiantes no lo perciben así" (Kieran, 1992).

Las ideas de concepción procedimental y estructural de una noción son acuñadas por Anna Sfard como dos formas de concebir una noción de matemáticas. En cuanto a la primera idea se refiere al reconocimiento que se hace de la existencia de una noción como potencial, es decir producto de acciones, en cambio en la concepción estructural la noción es referida como si tuviese existencia real y por lo tanto se reconoce y manipula a primera vista sin requerir detalles. Esta visión se remite también a la existencia de etapas históricas de la evolución de una noción en matemáticas y permite ver "el álgebra escolar como una serie de ajustes proceso-objeto que los estudiantes deben hacer para comprender el aspecto estructural del álgebra" (Kieran, 1992).

Es fundamental entonces que el docente de un primer curso de matemáticas en la universidad, examine las formas como usualmente ha aproximado a sus alumnos al estudio de una noción y cuáles son los acentos que se han dado a dicha aproximación. Este ejercicio requiere de una mirada profunda a los soportes de la cotidianidad de la labor docente, en cuanto se identifique (por cada uno) cuáles son los elementos orientadores de dicha labor. Por ejemplo, ¿un texto señala el camino de acción del docente? ¿Un programa de contenidos indica la ruta correcta de trabajo? ¿Las indicaciones e instrucciones de departamentos de matemáticas responsables de los cursos, imponen la forma de trabajo con el estudiante? Las respuestas permitirán reconocer cómo se conciben las actividades que usualmente se proponen como recursos de nivelación de conocimientos y habilidades en matemáticas para determinar sus posibles impactos en la transformación del nuevo estudiante.

Las actividades que posibiliten la reelaboración de una noción demandan una declaración de intencionalidad, en cuanto docente y estudiante puedan, a través de esta declaración, identificar qué acciones deben llevar a cabo para alcanzar los propósitos de sus cursos de matemáticas. Esta explicitación evitará que los supuestos sobre el posible impacto de una actividad guíen el quehacer del aula. Por ejemplo, se atacarían creencias acerca de la realización de un sinnúmero de ejercicios extraídos de diferentes textos, como soporte de comprensión de una noción. O se confrontarían convicciones de aprendizaje basadas en la visualización de la experticia del docente. En suma se puede esperar un desempeño tanto del docente como del estudiante, que consulte los intereses y responsabilidades de cada uno en el camino de la reelaboración de la noción en estudio.

- Construir la intencionalidad de una actividad de reelaboración de una noción básica de matemáticas, en un curso de matemáticas de primeros semestres, requiere una reflexión sobre los soportes del quehacer docente y sobre el papel que institucionalmente se asigna a la actividad. 
En este sentido es importante comprender y declarar que la tradición señala que el sistema de educación superior como todo el sistema escolar tiene un eje de acción: la enseñanza; es decir, se asume que el aprendizaje se deriva de ella y como tal las actividades denominadas de innovación, o las reformas escolares, se centran en mejorar la enseñanza con la expectativa que ello mejore el aprendizaje. Ahora, en las nuevas exigencias que la sociedad hace al sistema escolar, se ha declarado que el aprendizaje debe constituirse en el centro de las actividades de la escolaridad.

Los intentos de responder a este papel que se quiere asignar a la escolaridad, requieren esfuerzo, reflexión e indagar acerca de los elementos que posibiliten este tránsito de uno a otro eje de acción. Así se evitaría la tradicional adaptación que los miembros de la comunidad escolar asumen cuando de reformas a la escolaridad se trata.

En lo que se refiere a la práctica docente en cursos de matemáticas de primeros semestres, es preciso que se miren en detalle las creencias que usualmente soporta este ejercicio. Como creencias se asume lo que los maestros consideran debe ser, sin cuestionamiento ni explicación, con respecto a las formas de trabajo en el aula y las posibilidades de modificación de las mismas. De estas creencias forman parte entre otras, lo que el maestro piensa acerca del conocimiento matemático, del papel del individuo que aprende, del maestro y del cómo se aprende. (Ortiz Hurtado, 1999)

En este sentido se requiere el planteamiento de acciones que ayuden a superar creencias sobre el quehacer docente en matemáticas, que se aferran a la norma, o aquellas que se sustentan en una práctica determinada porque siempre ha sido así, porque así se ha enseñado tradicionalmente y así se ha aprendido.

Las acciones de las que se habla, deben posibilitarse desde una mirada a los elementos que constituyen la actividad matemática en un curso a los que se ha referido este escrito. Las concepciones que se tienen sobre: la matemática, el conocimiento matemático escolar, la función del docente, la evaluación, el papel del el estudiante, los recursos, deben ser expuestas claramente por el docente y contrastadas con los soportes de dichas concepciones, con el fin de visualizar posibles caminos de acción.

La tarea que se describe superficialmente es enorme; está mediada por problemáticas sociales que afectan la labor docente y corre el riesgo de no ser abordada adecuadamente, a menos que se identifiquen alternativas susceptibles de concretarse en la realidad que vive el docente en cuanto a su práctica profesional, en cursos de matemáticas de primeros semestres.

Dos alternativas son viables como forma de trabajo y pueden contribuir a la construcción de propuestas de actividades de reelaboración de nociones de matemáticas,

-La aproximación a la producción académica relacionada con las situaciones escolares de trabajo con las matemáticas. En este sentido, se ha generado un tipo de conocimiento denominado didáctica de las matemáticas. En particular se ha investigado ampliamente sobre tópicos relacionados con el álgebra escolar y los rudimentos del cálculo, los cuales parecen ser los temas centrales de los cursos de matemáticas en los primeros semestres de un programa de estudios universitarios.

La bibliografía existente provee información y abre expectativas de acción sobre aspectos relacionados con la enseñanza, con el aprendizaje, con el contenido propio de una temática, o con el impacto de la tecnología en la enseñanza y el aprendizaje de los tópicos mencionados.

-La incursión en el estudio a profundidad de las características que debe tener un sistema escolar que declare el aprendizaje como su centro de acción y de su influencia en la clase de matemáticas, o en el estudio de los soportes que posibiliten el tránsito de una escolaridad en matemáticas con base en la enseñanza a una que se soporte en el aprendizaje, o a la caracterización de una educación matemática centrada en el aprendizaje.

Una forma plausible de abordar las alternativas que se proponen, es la selección de una temática propia del álgebra escolar o de los rudimentos del cálculo y explorarla desde perspectivas señaladas por la didáctica de las matemáticas.

-Un aspecto esencial para abordar el trabajo de activida- 
des de re-elaboración de una noción en matemáticas es el institucional. De las definiciones y orientaciones que la institución universitaria señalen en cuanto a tiempos, espacios recursos y noción de calidad para cursos de matemáticas de primeros semestres, dependerá en gran medida que propuestas de solución a la problemática abordada tengan posibilidades reales de ejecución.

Las definiciones y orientaciones de que se habla antes, requieren a su vez de un marco de acción referido a las declaraciones que en cuanto a flexibilidad y cobertura sean asignadas por la institución a los cursos referidos. Respecto de la flexibilidad es preciso que la institución dé espacio para reconocer el estado real de los estudiantes que acceden a sus aulas, en cuanto a conocimientos previos en matemáticas y habilidades de pensamiento básicas. Esto posibilitará planteamientos de cursos que consulten este estado e intenten conciliarlo con las exigencias intelectuales para avanzar en el desarrollo de pensamiento superior. Así mismo generará una visión de un curso de matemáticas el cual no accione alrededor del cumplimiento rígido de un cronograma de contenidos, sino alrededor de actividades de reelaboración de nociones básicas en matemáticas.

En cuanto a la cobertura de un curso de matemáticas de estos primeros cursos, es importante que la institución indique en términos de procesos de formación de pensamiento superior cuales son susceptibles de desarrollarse desde tales cursos. Aquí hay una responsabilidad para los programas de estudio de la institución, la de formular los propósitos de incorporación de una formación básica en matemáticas dentro de sus mallas curriculares. Esta declaración es pertinente como elemento orientador de las propuestas de cursos de matemáticas y señalan rutas a explorar en conjunto por docente y estudiante en el camino de formación de este último.

Ahora, con un marco de referencia claro será posible que los responsables de la elaboración de planes de estudio de un programa, piensen en la determinación de tiempos mínimos exigibles a un estudiante para que alcance el nivel intelectual requerido para abordar con éxito los procesos propuestos en tales planes de estudio. Para el caso de los cursos de matemáticas, será factible una declaratoria de estos tiempos en términos de la obligación de reelaborar nociones fundamentales para su avance en el estudio de la disciplina. Aquí será de gran importancia catalogar el tiempo de estudio del estudiante como elemento primordial hacia el logro de objetivos propuestos. La especificación de tiempo de trabajo tanto en la clase como extraclase necesario para asumir la tarea propuesta, será clave para garantizar posibilidades de éxito al curso de matemáticas que se desarrolle. Así mismo proveerá elementos para asumir administrativamente el curso desde la perspectiva de créditos académicos.

La declaración de los espacios susceptibles de activarse con la propuesta de reelaboración de una noción en matemáticas, será crucial para alcanzar logros esperados. Espacios físicos (en el aula o fuera de ella) o virtuales, deben ser caracterizados por la institución para que sean susceptibles de ser usados por la comunidad universitaria en el trabajo conjunto que se prevé. La asignación de recursos también es factor vital para desarrollar los cursos de matemáticas desde las visiones antes señaladas.

Finalmente un factor determinante en el desarrollo de las actividades de reelaboración caracterizadas a lo largo del escrito, es el relacionado con lo que entiende la institución como calidad en la educación y formación matemática de sus estudiantes. Esta declaración debe servir como guía de evaluación del trabajo que se proponga para que un estudiante que ingrese a la universidad, desde cualquier nivel de formación en matemáticas dado, logre acceder a los requerimientos intelectuales mínimos necesarios para asegurar su formación básica en matemáticas. Y que logre realizar un tránsito por un programa de estudios que le permita alcanzar mejores comprensiones sobre el conocimiento abordado y luego le asegure un ejercicio profesional riguroso.

\section{A MANERA DE CONCLUSIÓN.}

La problemática que atraviesa el desarrollo de cursos de matemáticas de primeros semestres del sistema de educación superior, es susceptible de ser considerada desde una perspectiva integral, en cuanto los diversos estamentos que constituyen esta comunidad escolar asumen papeles de responsabilidad en la transformación de dichos cursos. 
La caracterización de una actividad de reelaboración de una noción en matemáticas provee un marco de referencia para construir propuestas que dinamicen los espacios asignados a cursos de matemáticas de primeros semestre, en una perspectiva de recuperación del estudiante para la actividad matemática, de replanteamiento de la práctica docente en matemática en un nivel superior y de la declaración de responsabilidades de la institución universitaria.

Se abren interrogantes acerca de elementos puntuales vinculados con las actividades de reelaboración y los cuales deben ser sujeto de estudio para configurar propuestas coherentes. Elementos de este orden son entre otros la evaluación, el contexto de acción de una noción en matemáticas, la validación del conocimiento que se tenga de una noción en matemáticas.

\section{BIBLIOGRAFIA}

-Cossio J y Tejada D. (1999). "Errores típicos en matemáticas de los estudiantes de primer semestre de la universidad" [en línea], disponible en: $<$ http://www2.unalmed.edu.co/dyna2005/128/errores.html>, consultado: 8 de julio de 2008.

-De Guzmán, M. "Matemáticas y estructura de la naturaleza." En F Mora y J Segovia de Arana. Ciencia y sociedad: Desafíos de conocimiento ante el tercer milenio. Oviedo. Fundación Santander Central Hispano, BSCH. Págs. $327-358$.

-GALLEGO, Rómulo. Saber pedagógico. Una visión alternativa. Bogotá: Cooperativa Editorial del Magisterio, 1992 .

-Kieran, C. ( 1992 ). "The learning and teaching of school algebra", disponible en: <http://dme.ufro.cl/pmat/images/Documentos/aprendizaje\%20del\%20algebra\%20en \%20e 1\%20liceo.pdf>, consultado: 5 de junio de 2008

-Lineamientos curriculares. Bogotá. Magisterio. 1998.

-Ortiz Hurtado, Myriam. La iniciación de la aritmética. Una propuesta de formación de profesores desde la perspectiva del aprendizaje. Tesis de doctorado. México. Departamento de Matemática Educativa del Centro de Investigación y de Estudios Avanzados del Instituto Politécnico Nacional de México. Doctorado en Matemática Educativa, 1999.

-Posso, A. (2005). "Sobre el bajo aprovechamiento en el curso de matemáticas I de la UTP" [en línea], disponible en: $\quad<$ http://www.utp.edu.co/php/revistas/ScientiaEtTechnica/docsFTP/164230169-174.pdf>,
Consultado: 8 de julio de 2008 .

-Robledo, J. (2005). "Formación matemática en un primer curso de matemáticas de la Universidad del Valle.”[en línea], disponible en: <http://www.icesi.edu.co/evenmat/ memorias/ConferenciaRobledo.pdf $>$, consultado: 8 de julio de 2008.

-Steen, Lynn. La enseñanza agradable de las matemáticas. México: Limusa. 2004

-TUNINGAMERICALATINA,(2003)."TuningEducational structures in Europe"[en linea], disponible en: $<\mathrm{http}: / /$ tuning.unideusto.org/tuningal/index.php?option $=$ com d ocman\&Itemid $=191 \&$ task $=$ view_category\&catid $=9 \&$ ord er $=$ dmdate_published\&ascdesc $=$ DESC $>$, consultado: 24 de junio de 2008.

-TUNING AMERICA LATINA, (2007). "Reflexiones y perspectivas de la Educación Superior en América Latina" [en línea], disponible en: <http://tuning.unideusto. org/tuningal/index.php?option $=$ com_docman\&Itemid $=$ $191 \&$ task $=$ view category $\&$ catid $=22$ \&order $=$ dmdate published \&ascdesc $=$ DESC $>$, consultado: 24 de junio de 2008.

- UNESCO, (2008). "Educación para todos en 2.015 ¿Alcanzaremos la meta?" [en línea], disponible en: <http:// unesdoc.unesco.org/images/0015/001548/154820s.pdf>, consultado: 21 de junio de 2008. 